\title{
Interactive comment on "Direct U-Pb dating of carbonates from micron scale fsLA-ICPMS images using robust regression" by Guilhem Hoareau et al.
}

\section{Guilhem Hoareau et al.}

guilhem.hoareau@univ-pau.fr

Received and published: 9 July 2020

RC1: "The paper by Hoareau et al. presents a new approach to U-Pb LA-ICPMS dating of carbonates that uses a mapping strategy in combination with robust regression of pixel $\mathrm{U}-\mathrm{Pb}$ ratios. The authors suggest the direct plotting of pixel values into isochron diagrams as a simple approach that allows easy visual access of data quality and can be applied in combination with pixel filtering or pixel colocalization. Imageguided or image based approaches to LA-ICP-MS dating of carbonate clearly have the advantage that some certainty about genetic homogeneity (or heterogeneity) of a sample can be gained. However, to collect a set of masses that are indicative of inclu- 
sions, alteration, or different generations of carbonate minerals in general requires a fast-scanning ICPMS instrument. The analytical setup used to acquire the data for the submitted study is not capable of fast mass scanning and thus no chemical information in addition to U-Th-Pb isotope data was collected. In order to still test for age homogeneity of the mapped portion of the sample, a pixel value colocalization approach is used. This allows to obtain information on the location and distribution of pixels representing outliers identified by plotting $\mathrm{U}-\mathrm{Pb}$ data in isochron diagrams and to draw subsets of the data for testing equivalence of the results".

Thank you for the positive comments. We clearly state in the initial manuscript that dating based on LA-ICP-MS isotope mapping is more suited to fast-scanning ICP-MS, so that the approach proposed by Drost et al. (2018) should be used (I. 247-248, for sample PXG20-1). Clearly, the colocalization study as we propose is an alternative way to select the pixels most amenable to precise dating. If fast-scanning ICPMS is available, we would suggest to first use chemical information to select a first area (Drost et al.), followed by our direct plotting of pixel values into diagrams and robust regression strategy to calculate the age.

$\mathrm{RC1}$ : "As advance in instrumentation and software allows or even requires exploring new strategies for data acquisition and processing, the presented approach seems to be a good addition to the methods currently employed. I am, however, not a statistician and can therefore not judge whether the statistical base is appropriately chosen and correctly applied to the presented workflow. The manuscript is mostly clearly structured and written but needs clarification in several points. The English language may need some polishing too."

Thank you for these mostly positive comments. The English language has been checked again.

RC1: "In the current version of the manuscript there is a lack of clarity on (1) how (or if) the data processing protocol deals with correction for instrumental drift, (2) how 
many NIST612 analyses are used for pixel ratio normalization (section 2.3.2; just the NIST analyses directly preceding and following that of the respective sample or those of the entire analytical session? And if the latter whether or not any excess scatter of the background- [and drift-?] corrected isotope ratios was observed) and (3) how the correction for radiogenic 208Pb from in-situ Th decay in Th-bearing samples is applied for the use of common 208Pb in the respective isochron plots. This should be clarified."

The comments proposed here are justified. In its first version, the text does not allow for a precise estimate of the way the corrections of isotopic ratios were made. To answer points 1) and 2): only isotopic maps of reference materials NIST 612 and WC1 analyzed just before and after the unknown have been considered. For pixel $\mathrm{Pb} / \mathrm{Pb}$ ratio of the unknown and of WC1, normalization (correction of mass bias) was performed using NIST 612 based on a standard bracketing approach, with the (robust) mean of NIST612 pixel values calculated as described in the text. The reference values of the NIST612 used for normalization for the lead ratios were taken from Woodhead and Hergt (2001), which was not specified in the main text. The drift-correction of the U/Pb ratios was also based on a standard bracketing approach, with (robust) mean $\mathrm{U} / \mathrm{Pb}$ value of the NIST612 preceding the unknown. This choice is the result of constraints related to the equipment available at the time of the study. Our home-made ablation cell cannot accommodate several samples simultaneously (unknowns + RMs) as it is commonly the case for commercial setups used in geochronology. Therefore, repeated RM analysis along with an unknown sample, and analysis of several unknowns (and associated RMs) during one session (for example, half a day) requires time-consuming sample handling and cannot be automated. As a result, our analysis workflow does not allow the calculation of an excess scatter by the repeated analysis of a homogeneous secondary standard, or by a pseudo-secondary standard approach based on Paton et al (2010) and as implemented in lolite ${ }^{\complement}$. Ideally, when future equipment will be available, we will move to a more traditional data reduction workflow based on repeated $\mathrm{RM}$ analysis, and the use of a dedicated software. To answer point 3): we agree

Interactive comment
Printer-friendly version

Discussion paper 
that this point has not been addressed in the manuscript, and will be added in the revised version. The followed method was similar to that proposed by Parrish et al. (2018), except that the ratios were calculated for each pixel rather than for individual spot analyses. The amount of radiogenic $208 \mathrm{~Pb}$ was calculated from measured $232 \mathrm{Th}$ and the age given by the T-W regression, allowing estimation of the amount of common $208 \mathrm{~Pb}(208 \mathrm{Pbc})$. The $208 \mathrm{Pbc} / 206 \mathrm{~Pb}, 206 \mathrm{~Pb} / 208 \mathrm{Pbc}$, and $238 \mathrm{U} / 208 \mathrm{Pbc}$ ratios are then straightforward to calculate. Given the low decay ratio of $232 \mathrm{Th}$, and the young age of most analyzed samples, variations in the value of the age chosen for calculation of radiogenic $208 \mathrm{~Pb}$ of up to $10 \mathrm{Ma}$ have negligible impact on the final age.

$\mathrm{RC} 1$ : "The identification of outlier pixel locations by colocalization is an attempt to data filtering that is not always efficient or successful. Apart from the usual "stray values" it may detect areas with low $\mathrm{U}$ and/or $\mathrm{Pb}$ concentrations where large scatter can exist due to insufficient counting statistics. Such scatter is inherent at very low ion signals and does not always justify exclusion of the data"

Indeed, a pixel filtering approach, whatever it is (trace element concentrations, isotope ratios, counting statistics, or colocalization) is not always perfectly efficient or successful. In the proposed approach, pixel selection based on colocalization in TeraWasserberg type diagrams must be seen as a guide to potentially use a spatial subset of the isotopic maps to get more precise (and hopefully more accurate) ages. This tool has clear limitations in the sense that it requires spending time analyzing isotope ratio images and it does not allow judging the origin of a possible dispersion of pixel values. In one of our examples (ARB), such scatter is indeed linked to a low $U$ concentration. Is the rejection of the corresponding area justified? We note that the filtering of pixels based on rejection of low counts for $238 \mathrm{U}$ (among other filtering approaches) has been proposed in the referential publication of Roberts et al. (2019), for the image-based dating of calcite sample NR1511. However, in our approach we do not reject pixels potentially distributed on several portions of the map; we select instead a map of lower area than the initial one, for which calculated ages are similar within the uncertainties,

GChronD

Interactive

comment
Printer-friendly version

Discussion paper

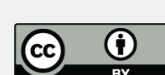


but more precise and with better statistics, than if the entire map is considered. This can be compared to the practice in LA-ICP-MS spot dating of carrying out several transects across a sample, and selecting the one giving the best results. An example is provided in Roberts et al. (2019) (their figure 7a), where the image-based choice of the transect is guided by higher $U$ concentration. This is equivalent to the rejection of transects corresponding to the lowest $U$ concentrations. finally, as stated several times in the manuscript (see above), colocalization was used because filtering based on trace element concentrations could not be performed with the available analytical set-up. We do not advocate the use of colocalization tooth and nail, but we suggest that it can be an interesting alternative (or better a complement) to the filtering approaches proposed elsewhere.

RC1: " (key words: limit of detection, limit of quantification)."

Based on NIST612, the limit of detection as of October 2019 was ca. 1.3 ppb and 0.2 $\mathrm{ppb}$ for $206 \mathrm{~Pb}$ and $238 \mathrm{U}$, respectively. The limit of quantification was ca. $4.3 \mathrm{ppb}$ and $0.8 \mathrm{ppb}$ for $206 \mathrm{~Pb}$ and $238 \mathrm{U}$, respectively. These values will be added to the revised version of the manuscript.

RC1: "The colocalization may also be successful in identifying areas with $U$ loss (when surrounded by areas with pristine $\mathrm{U}-\mathrm{Pb}$ systematics), where highly radiogenic $\mathrm{Pb}$ isotope compositions at low $238 \mathrm{U} / 206 \mathrm{~Pb}$ would occur. The colocalization technique is, however, likely to fail in a case where different types/generations of carbonate minerals yield data point arrays that are intersecting each other in a small angle resulting in largely overlapping data point arrays. Please see comments to sections 3.1.4 and 3.2.2 for further explanation."

We agree with this comment. See our answer below (dealing with sections 3.1.4 and 3.2.2)

RC1: "The lack of reported (numerical) data is also an issue. According to their data policy the journal requires a statement on where the data underlying a study can be 
found. Although the study presents/uses new data, there are no data submitted with the $\mathrm{ms}$ and a download link or a statement on data availability are missing. The approach presented in the study is new and associated data tables would be huge when choosing a format that would allow the reviewer/reader to plot and play with the data. Yet for potential further review and the case the manuscript is accepted for publication the authors should come up with an idea on data reporting and make the data accessible (Raw data files? Fully processed numerical pixel data? At the very least fully processed numerical values for sets obtained by discretization?...)."

We propose to add raw normalized $(\mathrm{Pb} / \mathrm{Pb}$ ratios) and drift-corrected $(\mathrm{U} / \mathrm{Pb})$ pixel data as supplementary material.

$\mathrm{RC1}$ : "Specific comments

Abstract: Reference citations are normally avoided in the abstract."

The abstract will be modified accordingly.

RC1: "1. Introduction: first paragraph - the authors may want to clarify that the very good spatial resolution allows to detect and exploit sub-millimetre scale heterogeneities in $\mathrm{U}$ and initial $\mathrm{Pb}$ concentration"

We agree. It will be clarified.

RC1: "second paragraph - "...spot ablations of sizes close to $100 \mu \mathrm{m} . .$. " this statement is too imprecise; my own quick literature survey revealed that spot diameters range at least from 80 to $235 \mu \mathrm{m}$ depending on lab and $\mathrm{U}$ concentration of the samples. The actual range of spot diameters should be used here and a few citations can be added to give somewhat more credit to previous work in LA-ICPMS U-Pb carbonate dating."

We agree, and propose to modify the sentence as follows: "Most carbonate dating LAICP-MS studies are based on spot ablations of sizes ranging from 80 to $235 \mu \mathrm{m}$ (e.g., Roberts and Walker, 2016; Parrish et al., 2018; Ring and Gerdes, 2016)" 
RC1: "third paragraph (from line 48) - it is not only detrital contamination that can be revealed by additional minor and trace element mapping but also alteration zones and GChronD different types or generations of carbonate minerals"

We agree, and propose to modify the sentence as follows: "However, in this case, the concentrations of elements characteristic of detrital contamination (e.g., $\mathrm{Zr}, \mathrm{Rb}$ etc), or allowing to locate alteration zones or distinct calcite cement generation (e.g., U, Pb, $\mathrm{Fe}, \mathrm{Mn}$ ) are used to define pixel exclusion thresholds. These are used to filter out the values corresponding to microscopic inclusions of other minerals (e.g., clays) and altered zones, and/or to isolate the cement generation targeted for dating"

$\mathrm{RC1}$ : "2.2.2 from Line 116: (i) lines of $50 \mu \mathrm{m}$ width separated by $100 \mu \mathrm{m}$ distance - this is confusing as it would leave 50 or $150 \mu \mathrm{m}$ wide gaps between lines but fig. S2 does not suggest there are gaps. Please rephrase."

To be more precise, the lines of $50 \mu \mathrm{m}$ height (not width) have been made every 100 $\mu \mathrm{m}$, leaving a gap of $50 \mu \mathrm{m}$ between them. On the preliminary isotopic maps of fig. $\mathrm{S} 2$, the lines corresponding to these gaps have been removed from the image to avoid a zebra-type rendering which made visualization difficult. Since this would result in images of height divided by 2 , the original aspect ratio was restored by stretching the image back to its original shape. Logically, the final pixels are therefore $100 \mu \mathrm{m}$ height instead of $50 \mu \mathrm{m}$. This will be specified in the figure caption.

RC1: "from Line 123: (ii) "...lines of $25 \mu \mathrm{m}$ width, separated by a distance of $25 \mu \mathrm{m} . . . "$ - again is this the spacing required by the "spot size" or are there $25 \mu \mathrm{m}$ gaps between the lines that are not ablated (fig. 1 suggests the former)"

The lines of $25 \mu \mathrm{m}$ height have been made every $25 \mu \mathrm{m}$, so that they are immediately adjacent to each other.

RC1: "from Line 128: is the preablation (and second map) run directly over the previous screening map or is the sample repolished between screening and dating experi-

Interactive

comment
Printer-friendly version

Discussion paper 
ments?"

The sample is repolished between the maps.

GChronD

RC1: "Line 130: the reference age of WC-1 is here given with $254.4 \pm 7 \mathrm{Ma}$ while Table 7.1 quotes $254.4 \pm 6 \mathrm{Ma}$. Why not just use the original age of $254.4 \pm 6.4 \mathrm{Ma}$ given by Roberts et al. (2017) for WC-1."

Interactive

comment

We agree, it will be corrected.

RC1: "2.3.2 Lines 148/149 " The standard error (i.e., standard deviation of the mean) for each ratio was also calculated from the standard deviation which is an output of the function." - is this referring to NIST612 analyses?"

Yes. It will be reminded in the revised manuscript.

RC1: "Line 153 - was an uncertainty applied when anchoring the regression? Roberts et al. (2017) give $0.85 \pm 0.04$ for WC-1."

There are currently no implementations of anchored regressions with uncertainties on the $y$-intercept in the $R$ language, which is used for robust regressions in our study. The same limitation arises when using IsoplotR (Vermeesch, 2018) for anchored York-type regressions.

RC1: "2.3.4 last sentence: The long-term excess variance cannot be derived from the primary reference material. Instead a quality control material (such as Duff Brown) should be used to assess this parameter."

Of course. It will be corrected.

RC1: "2.3.5 - Residual Standard Error - A short statement on the range of acceptable RSE for different signal intensities could be given as dispersion is not only ruled by age homogeneity of the sample but also by counting statistics. "

Concerning the Residual Standard Error, you raised a relevant question. Making the 
link between signal intensity and RSE would necessitate several tests on appropriate carbonate standards of known composition, with varying laser ablation intensity. This will be hopefully done in future analytical sessions. Our experience with analyzed carbonate samples is that successful samples all had an RSE lower than ca. 10-12\% of the $y$-intercept value (i.e., $<0.1$ for a common $\mathrm{Pb}$ value of 0.85 in Tera-Wasserburg diagrams), whatever the average concentrations in $U$ and $\mathrm{Pb}$. Note that the RSE allows a quick estimation of the scatter around the regression line (residuals), but good overall alignment of the points, which is a better estimate of age homogeneity, is more conveniently estimated with the d-MSWD and the running mean. Indeed, LA-ICP-MS spot analyses demonstrate that even scattered data can lead to meaningful ages, as soon as the MSWD reaches acceptable values.

$\mathrm{RC1}$ : “- discretizing of pixels into sets by increasing $238 \mathrm{U} / 206 \mathrm{~Pb}$ ratios - what are acceptable values for d-MSWD?"

A perfect alignment of the ellipses produced from the pixel value dataset will give a value of 1 for the d-MSWD, as for the MSWD in York-type regressions. Our empirical experience on analyzed samples shows that meaningful ages are obtained with d-MSWD lower than about 2.5, which is higher that the theoretical expectations for the critical MSWD values in York-type regressions, for more than 6 degrees of freedom (Wendt and Carl, 1991). However, contrary to the latter, there is currently no theoretical basis for the definition of isochrons versus errorchrons based on the d-MSWD, as reminded in section 5.2. We are currently working on a robust goodness-of-fit estimator for the robust regression approach, inspired by Powell et al. (2020).

$\mathrm{RC1}$ : "And wouldn't it be more appropriate to group the pixels into sets along the regression line rather than along the $x$-axis (i.e. $238 \mathrm{U} / 206 \mathrm{~Pb}$ ) as the data points are scattered in both the $\mathrm{x}$ - and $\mathrm{y}$-directions"

We agree and have changed the way of discretizing the pixel values. Note that the regression slopes are always close to 0 in the diagrams used for age calculation, which 
will not noticeably change the results compared to the former method.

RC1: "2.3.6 Lines 209/210: "...not necessarily more accurate..." - shouldn't this read "not necessarily more precise"?"

Yes. The mistake will be corrected.

RC1: "3.1 "... This is due to Pb0 values lower than that calculated from data of Hill et al. (2016) $(0.738 \pm 0.01,1 \mathrm{~s})$. The discrepancy between both values is explained by the high $238 \mathrm{U} / 206 \mathrm{~Pb}$ ratio of the sample (typically $60-70$ ) that prevents a precise determination of the common lead value. ..." It is not the high $238 \mathrm{U} / 206 \mathrm{~Pb}$ ratio or the low $207 \mathrm{~Pb} / 206 \mathrm{~Pb}$ intercept itself but rather the lack of spread of the data points along a linear array which results in a poorly defined slope of the regression line and thus in imprecise and inaccurate values for both intercepts. Please clarify."

You are entirely right. This is what we wanted to explain, but obviously it wasn't written clearly.

$\mathrm{RC1}$ : "Also an uncertainty for the initial $207 \mathrm{~Pb} / 206 \mathrm{~Pb}$ composition of Duff Brown is quoted but anchoring is done without uncertainty as suggested by fig. 2."

See our answer concerning the common lead value of WC1.

$\mathrm{RC1}$ : "3.1.2 Line 227: "..large variation in the $\mathrm{U} / \mathrm{Pb}$ ratios and a large amount of $\mathrm{U}$..." - both parameters could be specified by giving the approx. range of U/Pb ratios and an approximate $U$ concentration or the range of $U$ concentration in brackets Lines 233-235:" This high precision can be related to the very good visual and statistical parameters calculated for this regression (good point alignment, very low RSE, and low d-MSWD..." - again the values could be given here rather than the narrative description of these values (also applies to further sections)"

The numerical values will be added to the revised version.

$\mathrm{RC1}$ : "3.1.4 The pixel data show a lot more scatter around the regression line than 
the error ellipses of the spot data, i.e. the band in which the pixel data fall is much wider than a band covering the scattered (!) ellipses. This is not surprising but may be a problem. While spots were likely placed in a way not overlapping different textures (and thus error ellipses reflect the heterogeneity of the sample), the maps include all textural components and the pixel data represent a mixture of these different components. If there are small variations in ages and/or common $\mathrm{Pb}$ compositions for the different textural components this would not be obvious from plotting the pixel data as the different data point arrays are largely overlapping and may be intersecting each other in a small angle. The colocalization can be useful to locate areas that are rather heavily affected by for instance $U$ loss but will not necessarily be able to detect subtle differences in U-Pb systematics that result in slightly different slopes and intercepts of the regression lines of different components within the sample. One possibility to test for homogeneity might be dividing the map in squares/tiles with a size of a certain number of pixels (e.g., $125 \times 130 \mu \mathrm{m}$ or corresponding to $20 \mathrm{~s}$ ) and to then check the alignment of the resulting ellipses. This is just one thing that could be tried but I don't know if this is feasible with the software used."

We agree with the comment that the dispersion of pixel values is a problem, as indeed several generations of calcites with similar ages and/or common lead values could be erroneously confused. This strengthens the interest of a filtering approach as proposed by Drost et al (2018), which allows to clearly identify these generations on the basis of their composition. In that sense our colocalization can bring some help too. We note, however, that with a spot approach the problem raised is also possible; you write "While spots were likely placed in a way not overlapping different textures (and thus error ellipses reflect the heterogeneity of the sample)". All the problem lies in the "likely". It is also quite possible to mix phases with spots if an initial petrographic and geochemical study is not done properly, as for example two cements could be analyzed as one giving intermediate values. Nevertheless, we have implemented the requested test ("dividing the map in squares/tiles with a size of a certain number of pixels (e.g., $125 \times 130 \mu \mathrm{m}$ or corresponding to $20 \mathrm{~s}$ )") in our code to check for heterogeneity. This

\section{C11}

\section{Interactive}

comment
Printer-friendly version

Discussion paper

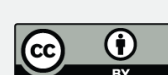


additional test will be now performed on a systematic manner. In the present study, the corresponding plots (represented in TW diagrams) will be added as supplementary data. The squares chosen have a dimension of $7 \times 6$ pixels, equivalent to $175 \mu \mathrm{m}$ $\mathrm{x} 150 \mu \mathrm{m}$ and 21 seconds of analysis, except for sample $\mathrm{BH} 14$ where they have a dimension of $4 \times 6$ pixels ( $100 \mu \mathrm{m} \times 150 \mu \mathrm{m}$; corresponding to $24 \mathrm{~s}$ ). Although using such low number of pixels for mean and standard error calculations result in rather large uncertainties (i.e., large ellipses), the results are conclusive for all samples. The ellipses obtained are always well aligned and York-type regression in TW diagrams give the expected ages, but with uncertainties higher than with the robust method even without propagation of systematic uncertainty. The MSWD are always lower than 1 , which is not surprising given the uncertainties of individual ellipses.

RC1: "3.2.1 Line 284: "more precise" rather than "more accurate"? It looks like about half of the data for cement $\mathrm{C} 2$ are squandered and not used for age calculation when selecting a rectangular area..."

We agree, the appropriate word is "precise". Concerning the chosen area, yes part of the isotopic map corresponding to cement $\mathrm{C} 2$ has not been used in this example. The idea was to show that even selecting a small part of the map could result in more precise ages than considering the entire map. Here, the subset was guided only by colocalization, rather than by petrographical analyses (and in the absence of trace element data, see lines 287-288). Combining both approaches shows that the subset belongs to cement $\mathrm{C} 2$ as identified from $\mathrm{CL}$ imaging.

RC1: "3.2.2 From the description in the text and the caption of figure 7 it is not clear to me which pixels were actually used for age calculation. The outlined area in the top left corner of the maps in figure 7 does however seem to have very low $\mathrm{U}$ and $\mathrm{Pb}$ concentrations and larger deviations of such data points from the regression line can be expected just from counting statistics."

We agree. The selected subset of the isotopic map corresponds to the entire map mi-

GChronD

Interactive

comment
Printer-friendly version

Discussion paper 
nus the top left corner. Although this selection was guided by colocalization, it appears that there is a strong link with $\mathrm{U}$ and $\mathrm{Pb}$ concentration, so scattered pixels are likely related with counting statistics.

RC1: "7.1 Analytical conditions table: There is a comment that "Ages in the data table are quoted at $95 \%$ absolute ..." I could not find a data table in the manuscript or supplement."

This is a mistake: ages in the figures, not in the data table.

RC1: "figure captions (figs. 2 - 5, 7): is "pseudo-ellipses" the intended term?"

We should have written "ellipses calculated from discretized pixel values".

RC1: "figures: Fonts in figures appear to be too small."

Their size will be increased as requested.

RC1: "figure S2: which element or isotope ratio is shown on the maps?"

These are the $238 \mathrm{U} / 206 \mathrm{~Pb}$ ratios. It will be specified in the caption.

Interactive comment on Geochronology Discuss., https://doi.org/10.5194/gchron-2020-10, 2020. 